\title{
Highlighting Significance of Weight Loss and Malnutrition in Older Adults
}

\author{
Vinod Nikhra* \\ Department of Medicine, Hindu Rao Hospital and NDMC Medical College, India
}

*Corresponding author: Vinod Nikhra, Senior Chief Medical Officer and Consultant, Department of Medicine, Hindu Rao Hospital and NDMC Medical College, New Delhi, India

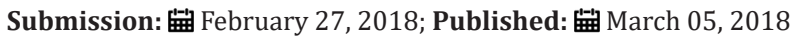

\section{Anorexia of Aging and Undernutrition}

There occurs a variable decrease in appetite in about $15 \%$ to $30 \%$ of older adults. This decline in desire to eat in older adults was described as the 'anorexia of ageing' by John Morley and Silver in 1988 [1]. The appetite deteriorates with increasing age, being higher in women, hospitalized patients, old age home residents and those living alone [2]. The reduced appetite and dietary intake is a harbinger of weight loss and malnutrition (WLM) leading to serious consequences for older adults. The weight loss in older adults may represent more of the tissue loss in skeletal muscle than adipose tissue. When excessive, it results in sarcopenia, often associated with protein-energy malnutrition and various deficiencies of vitamins and essential minerals.

\section{Incidence of WLM in Older Adults}

Body composition changes with age. Lean body mass begins to decrease up to $0.3 \mathrm{~kg}$ per year in the third decade, which is offset by gain in fat mass that continue until 65 to 70 years of age. Total body weight usually peaks at 60 years of age with a small decrease of 0.1 to $0.2 \mathrm{~kg}$ per year after 70 years of age. But, a significant weight loss in older adults is not a normal part of the aging process. In fact, it is related to general weakness, asthenia, decreased immunity, depression and an increased rate of disease complications and morbidity, and mortality. In fact weight loss of over 5 to 10 percent of body weight is a serious phenomenon, and unintentional weight loss of more than $5 \%$ within six to 12 months is associated with increased morbidity and higher mortality in older adults [3].

In a study it was documented that the institutionalized elderly patients who lost 5 percent of their body weight in one month were four times more likely to die within one year [4]. Also, in Alzheimer's disease patients, the weight loss correlates with disease progression, and a weight loss of over 5 percent is a significant predictor of deterioration of clinical features and elevated mortality [5].

\section{Pathophysiology of WLM in Older Adults}

The physiological anorexia of aging occurring in otherwise healthy older adults is possibly caused by changes in the digestive tract, gastrointestinal hormone release and activity, and neurotransmitters, and cytokines [6]. The animal studies have suggested that older rodents have an excessive satiety effect of cholecystokinin, and a decreased opioid feeding drive. Some older persons develop anorexia in association with depression, where excess corticotropin-releasing factor may be the neurotransmitter involved in the pathogenesis of the anorexia. In Alzheimer's disease, decreases in norepinephrine and neuropeptide Y may be involved in the anorexia [7].

An underlying disease and its treatment can directly cause anorexia and malnutrition, and the prevalence of WLM increases with the number of comorbidities. The most common causes are depression, malignancy, gastrointestinal disorders, renal disease, pulmonary disease, cardiac disorders (coronary heart disease and heart failure), dementia, alcoholism and several prescription medications. Various psychologic and social factors may also be involved. No cause is found in about one-fourth of patients. With aging, there occurs loss of lean body mass, a decrease in the basal metabolic rate and alterations in the senses of taste and smell. The decreased salivation and loss of teeth resulting in chewing difficulties also affect food intake.

\section{The Diagnosis and Clinical Evaluation}

The history may reveal information about the cause of WLM. A combination of various factors may be present. The dietary history and nutritional assessment are important, as are the Functional and mental status. A thorough review of medications may reveal the iatrogenic cause of altered taste, anorexia and digestive disturbances. Many individual medications have been associated with unintentional weight loss. Some selective serotonin reuptake inhibitors (SSRIs), such as fluoxetine, as can do sedatives and narcotic analgesics by interfering with cognition and the ability to eat. Psychosocial evaluation may discover some relevant issues. Appetite is strongly influenced by the environment and mood, and depression which commonly impairs appetite often afflicts the older adults. Retirement can also alter meal patterns and food choices due to change in routine, location, social contact and finances [8]. 
The physical examination in case of WLM is directed by the information gathered by the clinical history. Anthropometric measurements, like height and weight, and an assessment of cognitive function and mood are important. It is necessary to evaluate the oral cavity, gastrointestinal, respiratory and other systems. Recommended diagnostic tests include a fecal occult blood, complete blood count, basic metabolic panel, liver function tests, thyroid function tests, C-reactive protein levels, erythrocyte sedimentation rate, glucose measurement, lactate dehydrogenase measurement, and urinalysis. Chest radiography, abdominal ultrasonography, and a further advanced test like CT and MRI scan should also be considered [9].

\section{The Treatment to Reverse Anorexia and WLM}

The treatment of WLM aims to treat the underlying cause and may require a multidisciplinary team approach. A comprehensive approach will involve dietary changes, environmental modifications, nutritional supplements, flavour enhancers, and appetite stimulants. In diet modifications incorporating individual preferences, softer food consistency to accommodate for chewing or swallowing disabilities, and assisted feeding may help.

Treatment of depression may help in improvement of appetite and cause weight gain. Antidepressant, mirtazapine improves appetite and promotes weight gain. Dronabinol is a cannabinoid indicated for the treatment of anorexia with weight loss in AIDS patients, and may also be helpful in patients with Alzheimer's disease [10]. Megestrol is helpful in patients with AIDS or cancer [11]. Cyproheptadine is an antihistaminic and anti-serotoninergic drug that may improve appetite. Anabolic steroids and agents with anabolic properties like oxandrolone and ornithine may improve the muscular wasting [12].

It is important for the clinician to evaluate and monitor regularly the patient's nutritional status. Both the nonpharmacological as well as pharmacological therapy should be provided in light of the clinical condition and progress. The information should be shared with the patient and his or her relatives, all the health care workers and members from the multidisciplinary team. Finally, any new or changing signs and symptoms should be noted, and all available treatment options need to be explored and individualized.

\section{References}

1. Morley JE, Silver AJ (1988) Anorexia in the elderly. Neurobiol Aging 9(1): 9-16.

2. Malafarina V, Uriz Otano F, Gilguerrero L, Iniesta R (2013) The anorexia of ageing: physiopathology, prevalence, associated comorbidity and mortality. A systematic review. Maturitas 74(4): 293-302.

3. Holder K, Holder K (2014) Unintentional Weight Loss in Older Adults. Am Fam Physician 89(9): 718-722.

4. Ryan C, Bryant E, Eleazer P, Rhodes A, Guest K, et al. (1995) Unintentional weight loss in long-term care: predictor of mortality in the elderly. South Med J 88(7): 721-724.

5. White H, Pieper C, Schmader K (1998) The association of weight change in Alzheimer's disease with severity of disease and mortality: a longitudinal analysis. J Am Geriatr Soc 46(10): 1223-1227.

6. Soenen S, Chapman IM (2013) Body weight, anorexia, and undernutrition in older people. J Am Med Dir Assoc 14(9): 642-648.

7. Morley JE (2012) Undernutrition in older adults. Fam Pract 29(Suppl 1): i89-i93.

8. Alvarenga L, Kiyan L, Bitencourt B, et al. (2009) The impact of retirement on the quality of life of the elderly. Journal of the Nursing School of the University of Sao Paulo 43(4): 794-800.

9. Huffman GB (2002) Evaluating and Treating Unintentional Weight Loss in the Elderly. Am Fam Physician 65(4): 640-651.

10. Volicer L, Stelly M, Morris J, McLaughlin J, Volicer BJ, et al. (1997) Effects of dronabinol on anorexia and disturbed behavior in patients with Alzheimer's disease. Int J Geriatr Psychiatry 12(9): 913-919.

11. Yeh SS, Wu SY, Lee TP, Olson JS, Stevens MR, et al. (2000) Improvement in quality-of-life measures and stimulation of weight gain after treatment with megestrol acetate oral suspension in geriatric cachexia: results of a double-blind, placebo-controlled study. J Am Geriatr Soc 48(5): 485-492.

12. Alibhai SMH, Greenwood C, Payette H (2005) An approach to the management of unintentional weight loss in elderly people. CMAJ 172(6): 773-780

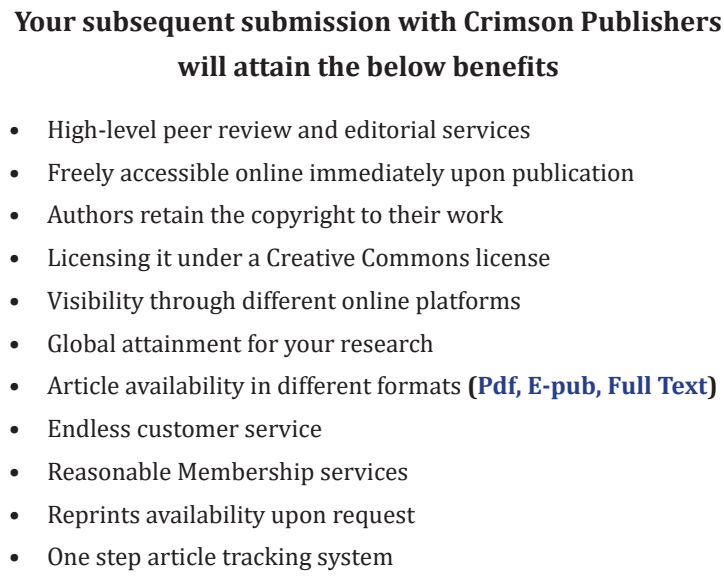

- High-level peer review and editorial services

- Freely accessible online immediately upon publication

- Authors retain the copyright to their work

- Licensing it under a Creative Commons license

- Visibility through different online platforms

- Global attainment for your research

- Article availability in different formats (Pdf, E-pub, Full Text)

- Endless customer service

- Reasonable Membership services

- Reprints availability upon request

- One step article tracking system 